\title{
A Comparison of Measured and Texture-Estimated Misorientation Distributions in Type 304 Stainless Steel Tubing
}

JUNWU ZHAO, BRENT L. ADAMS and PETER R. MORRIS Department of Mechanical Engineering, Brigham Young University, Provo, UT 84602, USA

(Received May 1, 1986; in final form May 20, 1987)

Dedicated to the memory of Professor Günter Wassermann

The Misorientation Distribution Function (MDF) was measured for commercial grade seamless 304 stainless steel tubing. Approximately 4000 boundary misorientations were used in the measurement. The results were compared with a theoretical MDF derived from the Crystallite Orientation Distribution Function (CODF) estimated from 1762 individual orientation measurements, and from x-ray pole-figure measurements, assuming grain orientations to be uncorrelated. It is concluded that the derived MDF can predict some features of the measured MDF but not all because of the presence of orientation correlations between adjacent grains.

KEY WORDS: Chanelling patterns, misorientation distribution function, random correlation, preferred correlation, series expansion, stainless steel.

\section{INTRODUCTION}

A new statistical measure of microstructure, called the Misorientation Distribution Function (MDF), was proposed by Bunge (1982) and reduced to practice by Haessner et al. (1983), and Pospiech $e t$ al. (1986). This function describes the probability that a grain boundary separates grains of misorientation $\Delta g$. Whereas the well- 
known Crystallite Orientation Distribution Function (CODF) provides useful input for property models which are insensitive to crystal boundaries, the MDF is useful in describing the structural distribution of grain boundaries themselves, and is therefore expected to be useful in predicting properties sensitive to boundary structure.

The importance of crystal boundary structure in the intergranular creep cavitation of stainless steel (Don and Majumdar, 1986) nickel (Lim and Raj, 1984), and iron-tin alloys (Watanabe, 1983) has recently been demonstrated. Comparisons of creep cavitation densities with the Coincidence Site Lattice (CSL) theory (Bollmann, 1970 ) show that creep cavitation is a strong function of $1 / \Sigma$-the fraction of coincident atoms sites formed by the two interpenetrating lattices across the boundary. Boundaries with large $1 / \Sigma$, such as $\Sigma 3$ twin boundaries, are most resistant to cavitation, whereas low $1 / \Sigma$ or random boundaries are damaged much earlier under creep conditions.

In a second example Hanada et al. (1986) have shown that $\Sigma 3$ boundaries in $\mathrm{Ni}_{3} \mathrm{Al}$ are very resistant to intergranular fracture. Since the specific energy varies for boundaries of differing $\Sigma$, we expect grain boundary structure to play a significant role in any process which creates free surface at the boundary, or otherwise changes boundary energy. The MDF provides a new measure of boundary structure which may prove useful in the study of intergranular phenomena.

It seems reasonable to inquire to what extent we might expect that the CODF is related to the MDF. The CODF gives the volume probability that grains have an orientation $g$ in the range $g, g+\Delta g$. The CODF is commonly estimated from a set of diffraction pole-figures, where intensities are collected by bathing a large number of grains $\left(10^{4}-10^{6}\right)$ simultaneously in the incident beam. Information concerning spatial arrangements of grains, or spatial correlations of grain orientations, is not available in experimental data obtained by this method. The assumption that grain orientations are spatially uncorrelated, implies a relationship between the CODF and the MDF.

In this paper we derive a theoretical expression for the MDF based upon the CODF when grain orientations are uncorrelated and certain other microstructural features are well behaved. 
Measured and theoretical MDFs are computed for commercialgrade seamless 304 stainless steel tube wall. The theoretical MDF is computed from a CODF estimated from 1762 individual-orientation measurements. The measured MDF is derived from the corresponding individual misorientation measurements for $\sim 4000$ boundaries. Both MDFs are represented in Euler space, using definitions similar to those of Pospiech et al. (1986). The results clearly demonstrate that the CODF can predict some features of the MDF but not all of them for 304 stainless steel. Furthermore, orientation correlations are expected to be important as more detailed structural measurements.

\section{REPRESENTATION OF THE MISORIENTATION DISTRIBUTION FUNCTION (MDF)}

Let $d S_{v}$ represent the grain boundary surface area per unit volume associated with the misorientation range $\Delta g, \Delta g+d \Delta g$. The Misorientation Distribution Function (MDF) is represented by $M(\Delta g)$ where

$$
\frac{d S_{v}}{S_{v}}=M(\Delta g) d \Delta g
$$

$S_{v}$ is the total grain boundary surface area per unit volume. The misorientation, $\Delta g$, is here represented by the set of three Euler angles $\alpha, \beta, \gamma$ which rotate the crystal reference frame $A$ into that of crystal $B$ as shown in Figure 1. Using this representation Eq. (1)

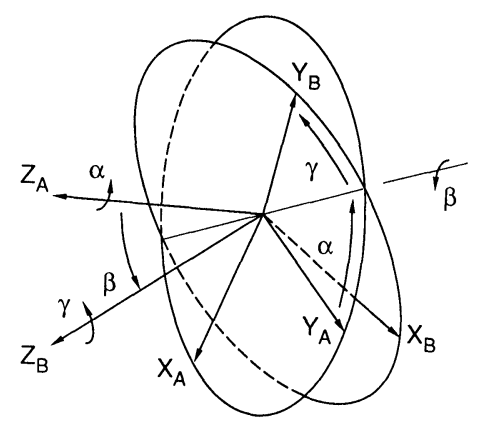

Figure 1 Euler angles $\alpha, \beta, \gamma$, which rotate crystal frame $A$ into crystal frame $B$. 
becomes

$$
\frac{d S_{v}}{S_{v}}=M(\alpha, \beta, \gamma) d(\cos \beta) d \alpha d \gamma
$$

We will represent the MDF with an infinite series of complete orthogonal functions in the space of Euler angles:

$$
M(\alpha, \beta, \gamma)=\sum_{l=0}^{\infty} \sum_{m=-l}^{l} \sum_{n=-l}^{l} M_{l m n} Z_{l m n}(\cos \beta) \mathrm{e}^{-i m \alpha} \mathrm{e}^{-i n \gamma}
$$

where $Z_{\text {lmn }}(\cos \beta)$ are the augmented Jacobi Polynomials defined by Roe (1965), and $M_{l m n}$ are series coefficients.

\section{A Symmetry considerations in the space of Euler angles}

We will restrict our analysis to cubic-crystal symmetry, where the crystal lattice can be rotated into 24 equivalent orientations. These 24 rotations form a group $L$ (see for example Hansen et al., 1978). If crystal $A$ is in orientation $g_{A}$ then there will be 24 equivalent orientations of $A, g_{A}^{e}$ given by $g_{A}^{e}=l_{i} \cdot g_{A}$ where $l_{i} \in L$. The same symmetry will apply to crystal $B$ which is misoriented from crystal $A$ by $\Delta g .\left(g_{B}=\Delta g \cdot g_{A}\right)$. It follows that

$$
g_{B}^{e}=l_{i} \Delta g l_{j} g_{A}
$$

or $\Delta g^{e}=l_{i} \Delta g l_{j}$.

Equation (4) requires that $M(\Delta g)=M\left(l_{i} \Delta g l_{j}\right)$, where $i, j=$ $1,2, \ldots, 24$. For the symmetry conditions noted, it may be shown (Roe, 1965), that

$$
\begin{aligned}
M_{l m n}= & M_{l m n}^{*}(\text { coefficients are real) } \\
M_{l m n}= & 0 \text { unless both } m \text { and } n \text { are integral } \\
& \text { multiples of } 4 \text { (including } 0) \\
M_{l m n}= & (-1)^{l} M_{l \bar{m} n} \\
M_{l m n}= & (-1)^{l} M_{l m \bar{n}} \\
M_{l m n}= & M_{l \bar{m} \bar{n}}
\end{aligned}
$$

Incorporating the relations given in (5), together with those given 
by Morris and Koskenmaki (1977) for the $Z_{l m n}(\xi)$ into Eq. (3) yields

$$
\begin{array}{r}
M(\alpha, \beta, \gamma)=2 \sum_{l=0}^{\infty} \sum_{m=0,4}^{\leq l} \sum_{n=0,4}^{\leq l}\left[1+\delta_{m}^{0}\right]^{-1}\left[1+\delta_{n}^{0}\right]^{-1} M_{l m n} \\
\left\{\left[Z_{l m n}(-\xi)+Z_{l m n}(\xi)\right] \cos (m \alpha) \cos (n \gamma)\right. \\
\left.+\left[Z_{l m n}(-\xi)-Z_{l m n}(\xi)\right] \sin (m \alpha) \sin (n \gamma)\right\}
\end{array}
$$

where $\delta_{m}^{0}, \delta_{n}^{0}$ are Kronecker delta functions.

The MDF must also exhibit symmetry with respect to the interchange of $A$ and $B$. Therefore it must be true that

$$
M(\Delta g)=M\left(\Delta g^{-1}\right)
$$

Equations (6) and (7) are consistent if $M_{l m n}=M_{l n m}$. The expression given in Eq. (6) satisfies only the tetragonal portion of the crystal symmetry exhibited by cubic (432) lattices. The symmetry introduced by the presence of four 3-fold rotation axes is not included. Roe (1966) has shown that the 3-fold rotational symmetry in cubic crystals gives rise to linear dependencies among the $M_{l m n}$ coefficients of the form

$$
M_{l m n}=U_{l n} M_{l m 0}+V_{l n} M_{l m 4} \quad(l \leq 20)
$$

where the real coefficients $U_{l n}$ and $V_{l n}$ has been tabulated by Roe (1966) for even $l$ to order 22, by Morris (1982) for odd $l$ to order 23, and by Esling et al. (1981) for even and odd $l$ to order 34 . Incorporation of relations in Eq. (8), and those following Eq. (7) into Eq. (6) reveals that only a few $M_{l m n}$ coefficients are independent; for example, only 6 coefficients are independent to $l=10$ in the expansion (6).

\section{B Calculation of $M_{I m n}$ coefficients for single boundary elements}

Generally we will measure the segment lengths observed in a planar cross section through grain boundaries, and the crystallographic misorientation across those boundary segments. This information can then be used to estimate the $M_{l m n}^{0}\left(\Delta g^{0}\right)$ which represents a structure with only a single misorientation, $\Delta g^{0}$, present. Therefore $M(\Delta g)=0$ unless $\Delta g=\Delta g^{0}$. Next, successively multiply both sides 
of the series expansion, Eq. (6) by the expression which gives the angular dependence for each independent coefficient, $M_{l m n}$; then integrate over the entire range of Euler space.

The method outlined here is detailed more completely by Morris (1983) for the CODF. The result is that we obtain expressions for the ideal coefficients $M_{l m n}$ in terms of the individual ideal misorientation angles $\alpha^{0}, \beta^{0}, \gamma^{0}$. For example

$$
\begin{aligned}
M_{400}^{0}= & \left\langle Z_{400}\left(\xi^{0}\right)+2(0.5976143) Z_{404}\left(\xi^{0}\right)\left[\cos 4 \alpha^{0}+\cos 4 \gamma^{0}\right]\right. \\
& +2(0.5976143)^{2}\left\{\left[Z_{444}\left(-\xi^{0}\right)+Z_{444}\left(\xi^{0}\right)\right] \cos 4 \alpha^{0} \cos 4 \gamma^{0}\right. \\
& \left.\left.+\left[Z_{444}\left(-\xi^{0}\right)-Z_{444}\left(\xi^{0}\right)\right] \sin 4 \alpha^{0} \sin 4 \gamma^{0}\right\}\right\rangle /\left\langle\left\{4 \pi^{2}\right\}\right. \\
& \left.\times\left\{1+4[0.5976143]^{2}\left[1+(0.5976143)^{2}\right]\right\}\right\rangle
\end{aligned}
$$

where $\xi^{0}=\cos \beta^{0}$.

For a set of $N$ discrete boundary misorientation measurements we may then estimate the coefficients of the MDF to be given by

$$
M_{l m n}=\sum_{i=1}^{N} L^{i} M_{l m n}^{i} / \sum_{i=1}^{N} L^{i}
$$

where $L^{i}$ is the length of the measure grain boundary in the section.

\section{A THEORETICAL MDF DERIVED FROM TEXTURE}

We wish to address the question concerning to what extent we may expect texture to yield information about the MDF. It is possible to derive a texture-based MDF, here denoted as $M^{\prime}(\Delta g)$, from the crystallite orientation distribution function (CODF) if two assumptions are made. These are:

i) Crystal orientations are uncorrelated with respect to spatial position.

ii) The grain boundary surface area per unit volume is distributed in orientation space in the same manner as the crystal volume.

The first of these assumptions means that if we consider any two points in the volume of interest and measure their respective orientations, the probability of one of these points having orientation $g$ with respect to a chosen reference co-ordinate system, and 
the other having orientation $g^{\prime}=\Delta g \cdot g$ is given by the expression

$$
\begin{aligned}
\frac{d V}{V}(g) \cdot \frac{d V}{V}\left(g^{\prime}\right) & =\frac{d V}{V}(g) \cdot \frac{d V}{V}(\Delta g \cdot g) \\
& =f(g) \cdot f(\Delta g \cdot g) d g d \Delta g
\end{aligned}
$$

where $f(g)$ is the Crystallite Orientation Distribution Function (CODF). Under the condition of assumption (i) we would find the same probability of occurrence for any choice of two points in the volume.

The second assumption places some restrictions on the microstructure of the polycrystals which are nominally satisfied for equiaxed grain structures. If we partition the grain-boundary surface area per unit volume according to orientation $g$, remembering that every boundary element is shared by two neighboring grains, then assumption (ii) requires that

$$
\frac{d S_{v}(g)}{S_{v}}=\frac{d V(g)}{V}
$$

where $d S_{v}(g) / S_{v}$ is the area fraction per unit volume of grain boundary associated with grains of orientation $g$ in the range $g$, $g+d g$. Equation (12) has the meaning that the grain-boundary area to grain-volume ratio is equivalent for grains of any orientation in an average sense.

If we combine the assumption of uncorrelated orientation with Eq. (12) we can express a new texture-derived MDF as the integral over all orientations, $g$, of the joint probability of a grain boundary sharing grains of orientation $g$ and $\Delta g \cdot g$. Thus we define $M^{\prime}(\Delta g)$ as

$$
\begin{aligned}
M^{\prime}(\Delta g) d \Delta g & =\left[\iiint_{g} \frac{d S_{v}(g)}{S_{v}} \cdot \frac{d S_{v}(\Delta g \cdot g)}{S_{v}}\right] \\
& =\left[\iiint_{g} f(g) \cdot f(\Delta g \cdot g) d g\right] d \Delta g
\end{aligned}
$$

The meaning of $M^{\prime}(\Delta g)$ is clear only when both the assumptions (i) and (ii) are satisfied. For an equiaxed-grain structure assumption (ii) is satisfied to first order. A comparison of $M^{\prime}(\Delta g)$ with the measured function, $M(\Delta g)$, will help us evaluate the reasonableness 
of assumption (i). This is of central importance since the CODF is much more accessible than the single orientation measurements required for the MDF.

We now represent the CODF, $f(g)$, as the usual series expansion. That is,

$$
f(g)=\sum_{l=0}^{\infty} \sum_{m=-l}^{l} \sum_{n=-l}^{l} W_{l m n} Z_{l m n}(\cos \theta) \mathrm{e}^{-i m \psi} \mathrm{e}^{-i n \phi}
$$

where $\psi, \theta, \phi$ are defined by Roe (1965). The series expansion for $f(\Delta g \cdot g)$ can be written in terms of the same Euler angles $\psi, \theta, \phi$ in Eq. (14) if we use Roe's rotation formula (1965) to rotate the final crystal frame at $\Delta g \cdot g$ to a crystal frame at $g$ by using the inverse rotation $\Delta g^{-1}$. Therefore

$$
\begin{aligned}
& f(\Delta g \cdot g)=\sum_{l^{\prime}=0}^{\infty} \sum_{m^{\prime}=-l^{\prime}}^{l^{\prime}} \sum_{n^{\prime}=-l^{\prime}}^{l^{\prime}} W_{l^{\prime} m^{\prime} n^{\prime}}^{\prime} Z_{l^{\prime} m^{\prime} n^{\prime}}(\cos \theta) \mathrm{e}^{i m^{\prime} \psi} \mathrm{e}^{-i n^{\prime} \phi} \\
& W_{l^{\prime} m^{\prime} n^{\prime}}^{\prime}=\left(\frac{2}{2 l^{\prime}+1}\right)^{1 / 2} \sum_{p=-l}^{l} W_{l^{\prime} m^{\prime} p} Z_{L^{\prime} p n^{\prime}}(\cos \beta) \mathrm{e}^{i p(\pi-\gamma)} \mathrm{e}^{i n^{\prime}(\pi-\alpha)}
\end{aligned}
$$

Introducing Eqs. (14-16) into Eq. (13) and using the properties

$$
\begin{gathered}
\int_{0}^{2 \pi} \mathrm{e}^{i m \psi} \mathrm{e}^{-i m^{\prime} \psi} d \psi=2 \pi \delta m m^{\prime} \\
\int_{0}^{2 \pi} \mathrm{e}^{i n \phi} \mathrm{e}^{-i n^{\prime} \phi} d \phi=2 \pi \delta n n^{\prime} \\
\int_{0}^{\pi} Z_{l m n}(\cos \theta) \cdot Z_{l^{\prime} m n}(\cos \theta) \sin \theta d \theta=\delta_{l l^{\prime}} \\
Z_{l \bar{m} \bar{n}}(\cos \theta)=(-1)^{m+n} Z_{l m n}(\cos \theta) \\
W_{l \bar{m} \bar{n}}=(-1)^{m+n} W_{l m n}^{*}\left({ }^{*} \text { implies complex conjugate }\right)
\end{gathered}
$$

we obtain

$$
\begin{aligned}
M^{\prime}(\Delta g)=4 \pi^{2} & \sum_{l=0}^{\infty} \sum_{m=-l}^{l} \sum_{n=-l}^{l} \sum_{p=-l}^{l}\left(\frac{2}{2 l+1}\right)^{1 / 2} \\
& \times W_{l m n}^{*} W_{l m p} Z_{l p n}(\cos \beta)(-1)^{p} \mathrm{e}^{-i p \gamma}(-1)^{n} \mathrm{e}^{-i n \alpha}
\end{aligned}
$$

Now separate $W_{l m p}$ into its real and imaginary components, 
$A_{\text {lmp }}+i B_{\text {lmp }}$, and consider that for crystallites of cubic symmetry $n$ and $p$ must be integral multiples of 4 for non-zero $W_{l m p}$, and also that

$$
\begin{gathered}
A_{l m \bar{p}}=A_{l m p}, \quad B_{l m \bar{p}}=B_{l m p} \\
Z_{l \bar{p} \bar{n}}(\cos \beta)=Z_{l p n}(\cos \beta) \\
Z_{l \bar{p} n}\left(\cos \beta=(-1)^{l} Z_{l p n}(\cos [\pi-\beta])\right. \\
Z_{l p \bar{n}}(\cos \beta)=(-1)^{l} Z_{l p n}(\cos [\pi-\beta])
\end{gathered}
$$

It is then possible to write Eq. (17) as

$$
\begin{array}{r}
M^{\prime}(\Delta g)=8 \pi^{2} \sum_{l=0}^{\infty}\left[\frac{2}{2 l+1}\right]^{1 / 2} \sum_{n=0,4}^{\leq l}\left[1+\delta_{n}^{0}\right]^{-1} \sum_{p=0,4}^{\leq l}\left[1+\delta_{p}^{0}\right]^{-1} \\
D_{l p n}\left\{Z_{l p n}(\cos \beta) \cos (p \gamma+n \alpha)+(-1)^{l} Z_{l p n}(\cos [\pi-\beta])\right. \\
\times \cos (p \gamma-n \alpha)\}
\end{array}
$$

where

$$
D_{l p n}=\sum_{m=-l}^{l}\left(A_{l m p} A_{l m n}+B_{l m p} B_{l m n}\right) .
$$

It is easy to demonstrate that $D_{l p n}$ has the same symmetry as $M_{l m n}$ given in Eq. (5) if the $W_{l m n}$ coefficients have cubic symmetry. Notice that when $l$ is even Eqs. (6) and (18) are identical when we set

$$
M_{l m n}^{\prime}=4 \pi^{2}\left(\frac{2}{2 l+1}\right)^{1 / 2} D_{l m n}
$$

The index $l$ is required to be even due to Friedel's (1913) law whenever pole figures are used to determine the CODF. Also note, however, that $D_{l m n}=D_{l n m}$, which leads to the conclusion that the coefficients $D_{l m n}$ are essentially the same as $M_{l m n}$ [when assumptions (i) and (ii) hold] when normalized by a factor of $4 \pi^{2}\{2 /(2 l+1)\}^{1 / 2}$. Equation (19) allows a direct comparison of the measured and CODF-derived MDF's.

The CODF-derived MDF can also be derived using the symmetric-function formalism of Bunge (1982). The resulting notation is more compact and in some respects it is more lucid. The corresponding development in Bunge's notation is given in the appendix. 


\section{EXPERIMENTAL PROCEDURES}

Selected-area channelling patterns, obtained using a JOEL 100CX electron microscope, provided the required individual-orientation measurements. Samples were cut from the wall of commercial-grade seamless 304 stainless steel tube. Test samples were heated to $1127^{\circ} \mathrm{C}$ for 0.5 hours to allow the grains to grow to an average size of $\sim 70 \mu \mathrm{m}$. In order for carbides to stabilize on the grain boundaries samples were aged for 72 hours at $775^{\circ} \mathrm{C}$ in an argon atmosphere. Samples were then mechanically polished on 400,600 , $5 \mu \mathrm{m}$ and $0.3 \mu \mathrm{m}$ grit polishing wheels. To reveal the grain boundaries and prepare the surface for microscopy, the samples were electro-polished with $10 \%$ perchloric acid, $70 \%$ ethanol, $15 \%$ water, and $5 \%$ butyl cellusolve for 15 to 20 seconds at an approximate current density of $0.5 \mathrm{amps} / \mathrm{cm}^{2}$. The channelling patterns for 1762 grains were measured and subsequently analyzed using the software developed by P. Heilmann et al. (1982). Approximately 4000 boundary misorientations were measured.

In order to compute the crystallite orientation distribution function (CODF) from individual orientation measurements, a mesh was generated to cover the grain boundary maps (about 70 points in an average grain section). The points of the mesh can be
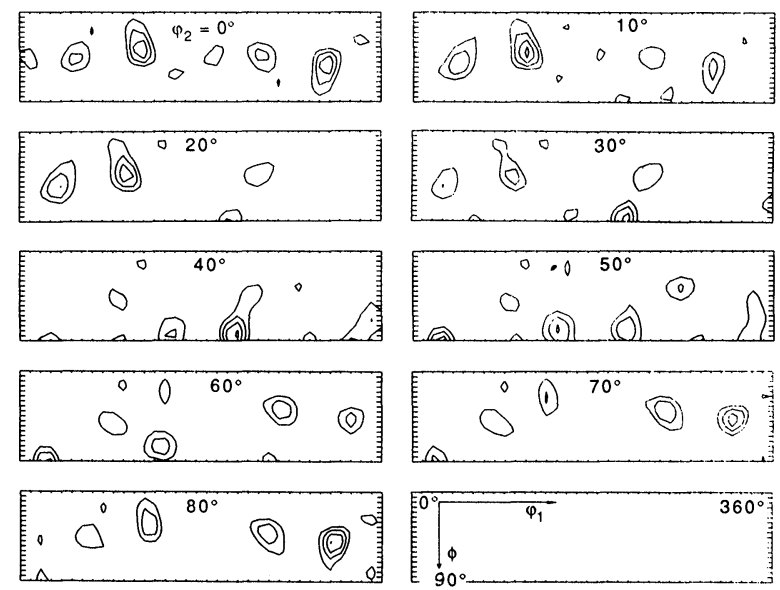

Figure 2 CODF determined from 1762 individual grains. Contour lines are in units of $1.5,2,2.5,3$, and 3.5 times random. 

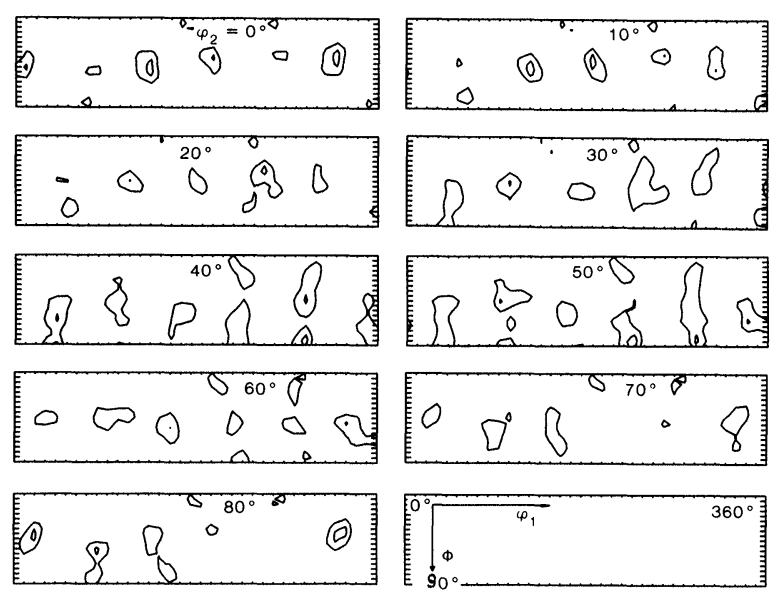

Figure 3 CODF determined from x-ray pole figure measurements. Contour lines are in units of 1.5 and 2 times random.

defined by a location in the sampling section and an orientation that is associated with the grain that the point lies in. The CODF was determined by calculating the series-expansion coefficients for each individual-grain orientation (the series expansion was truncated at 16th order). The coefficients were weighted by the number of points in the grain and then summed to determine the coefficients for the whole data set. This CODF is shown in Figure 2 using contour plots in the Euler angles defined by Bunge (1982). The series-expansion coefficients were used to calculate the texture-estimated misorientation distribution function.

$\mathrm{X}$-ray pole-figures were also determined using $\mathrm{MoK}_{\alpha}$ radiation. $\{111\},\{110\}$, and $\{200\}$ pole-figures were used to calculate the CODF using the method of Bunge (1982) to the same order in the series expansion $(l \leqslant 16)$. The results are shown in Figure 3.

\section{RESULTS AND CONCLUSIONS}

Comparison of the single-orientation determined CODF (Figure 2) with the x-ray determined CODF (Figure 3) gives some confidence that the set of single orientation measurements is substantially 
representative of the bulk texture. Most of the peaks present are common to both CODF's, although shifts of $\sim 10^{\circ}$ are noted in some cases. Shifts of this magnitude are not unexpected in view of the theoretical resolution of limited single-orientation measurements, which is estimated to about $16^{\circ}$ for this data set (Adams et al. 1987).

Measured and CODF-derived misorientation-distribution functions are shown in Figures 4 and 5. They are plotted in constant- $\beta$ sections through the range of misorientation space given by $0 \leq \alpha \leq \pi / 2, \quad 0 \leq \cos \beta \leq 1 / \sqrt{ } 3, \quad 0 \leq \gamma \leq \pi / 2$. Peak contours have been normalized to times-random values. The domain chosen is six-fold redundant. Similar redundancy problems occur when the rotation axis and rotation angle are chosen to represent misorientation instead of Euler angles (Haessner et al., 1983, Pospiech et al., 1986).

The prominent feature of the measured MDF is the strong peak centered on the $\Sigma 3$ (angles $\alpha=45, \beta=70.53, \gamma=45$ ) and the secondary peak located at $\Sigma 9$ (angles $\alpha=63.55, \beta=83.73, \gamma=$ 63.66) positions. The intersection of two twins result in boundaries of either $\Sigma 1$ or $\Sigma 9$ character (Don and Majumdar, 1986). It is

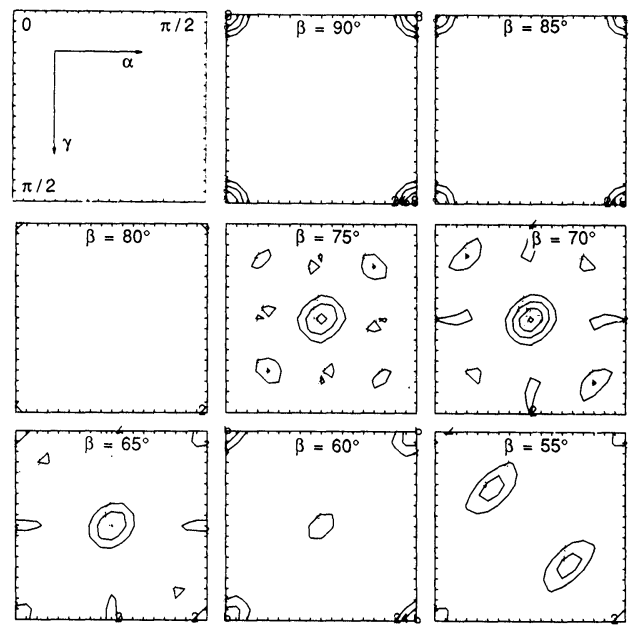

Figure 4 Sixteenth-order expansion of experimentally determined Misorientation Distribution Function. Level lines are in units of times random. 


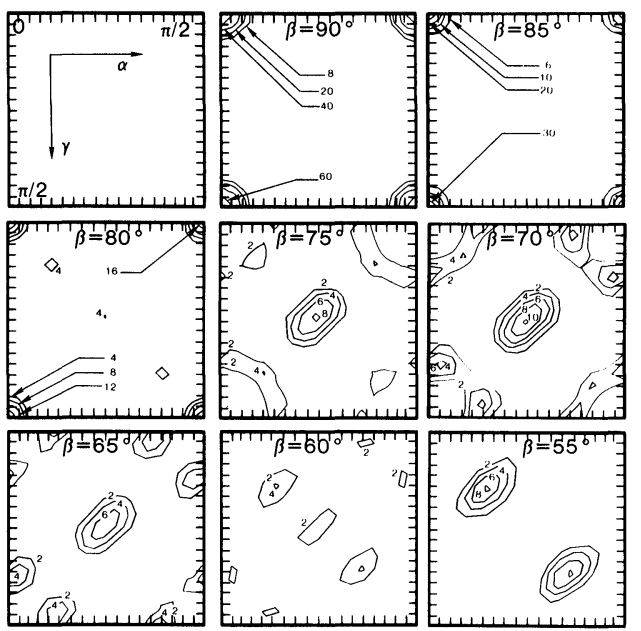

Figure 5 Sixteenth-order expansion of Misorientation Distribution Function calculated from Crystallite Orientation Distribution Function. Level lines are in units of times random.

apparent that this feature is also present in the CODF-based MDF although the level values differ in magnitude. Both measured and CODF-based MDFs have strong peaks in the $\Sigma 1$ (angles $\alpha=0$, $\beta=90, \gamma=0$ ) position. In the measured function this result reflects the low-angle character of some apparent grain boundaries, the theoretical MDF artificially predicts that $\Sigma 1$ boundaries will occur as a consequence of the assumption of perfect disorder among crystallites at near-neighbor distances.

Some peaks in the texture-based MDF do not show in the measured MDF. For example, peaks such as that observed at $\alpha=65, \beta=70, \gamma=5$ in the theoretical MDF are not observed in the experimental MDF. This suggests the existence of some degree of orientation coherence in the structure. Orientation coherence may be expected, due to the variation of grain-boundary energy with intercrystallite misorientation. The CSL theory (Bollmann, 1970) provides a geometric basis for the existence of special, low energy boundaries in polycrystals, but recent experimental evidence (Maurer and Gleiter, 1979) suggests that electronic structure may be more important than geometric coincidence in the noble metals. 
The present study provides evidence of the prevalence of non-CSL boundaries, such as those associated with $\alpha=30, \beta=55, \gamma=30$, in the experimental data.

We have previously compared our measured data with a set measured by Don and Majumdar (1986) for a rod sample of type 304 stainless steel (Zhao and Adams, 1986). The measured MDF's were found to be of essentially the same character, but with some higher values in the $\Sigma 3$ position in Don's data. Essentially half of all boundaries were found to have $\Sigma 3$ character in both the type 304 stainless steel investigated by Don and Majumdar (1986), and the nickel studied by Lim and Raj (1984). In the 304 stainless steel, the observed boundaries do not appear to have twin character, but presumably they are remnants of earlier annealing twins. It is believed that twin boundaries are highly mobile in these materials.

We conclude that CODF can predict some features of the MDF in type 304 stainless steel under the assumption of spatial noncorrelation of texture. However, more detailed measurements of microstructure are necessary than the present measurement of the CODF. These measurements must contain a spatial dependence in order to properly account for the presence of orientation coherence. The development of the orientation-coherence function (OCF) has been considered by Kroner (1972), Plege (1986) and Adams et al. (1987). The texture-based MDF defined in Section III, must give some information about the far-field misorientation correlation.

\section{Acknowledgements}

J. Zhao and B. L. Adams wish to acknowledge the support of the office of Basic Energy Sciences, U.S. Department of Energy under grant number DE-FG0285ER 45203. The assistance of Dr. Jarlen Don of Southern Illinois University and Dr. Nestor Zaluchek and other members of the staff of the electron Microscopy Center at Argonne National Laboratory is gratefully acknowledged.

\section{References}

Adams, B. L., Morris, P. R., Wang, T. T., Willden, K. S. and Wright, S. I., "Description of Orientation Coherence in Polycrystalline Materials," submitted to Acta Metall. January 1987.

Bollmann, W. (1970). Crystal Defects and Crystalline Interfaces, Springer-Verlag, New York.

Bunge, H. J. (1982). Texture Analysis in Materials Science, Butterworth, London. 
Don, J. and Majumdar, S. (1986). Acta Metall. 34, 961.

Esling, C., Bechler-Ferry, E. and Bunge, H. J. (1981). "ICOTOM6, The Sixth International Conference on Textures of Materials," Tokyo, Japan, Vol. 2, 1373-88. Iron and Steel Institute of Japan.

Friedel, G. (1913). Compt. rend. 157, 1533-6.

Haessner, F., Pospiech, J. and Sztwiertnia, K. (1983). Materials Science and Engineering. 57, 1-15.

Hanada, S., Ogura, T., Watanabe, S., Izumi, O. and Masumoto, T. (1986). Acta Metall. 34, 13-21.

Hansen, J. Pospiech, J. and Lucke, K. (1978). Tables for Texture Analysis of Cubic Crystals, Springer-Verlag, Berlin.

Heilmann, P., Clark, W. A. T. and Rigney, D. A. (1982). Ultramicroscopy 9, 365.

Kroner, E. (1972). Statistical Continuum Mechanics, Springer-Verlag, Wien.

Lim, L. C. and Raj. R. (1984). Acta Metall. 32, 1177-81.

Maurer, R. and Gleiter, H. (1979). Scripta Met. 13, 61.

Morris, P. R. (1982). Textures and Microstructures 4, 241-2.

Morris, P. R. (1983). Textures and Microstructures 5, 191-204.

Morris, P. R. and Koskenmaki, D. C. (1977). Texture of Crystalline Solids 2, 253.

Plege, B. (1986). Experimental Techniques of Texture Analysis. Ed.: H. J. Bunge. DGM Informationsgesellschaft Oberursel, 315-328.

Pospiech, J., Sztwiertnia, K. and Haessner, F. (1986). Textures and Microstructures. 6, 201.

Roe, R. J. (1965). J. Appl. Phys. 36, 2024-31.

Roe, R. J. (1966). J. Appl. Phys. 37, 2069-72.

Watanabe, T. (1983). Metall. Trans. 14A, 531.

Zhao, J. and Adams, B. L. (1986). Prof. Int. Conf. Fatigue, Corrosion Cracking, Fracture Mechanics, and Failure Analysis: the Mechanism of Fracture, ed. V. S. Goel, American Society for Metals (1986) p. 597-603.

\section{APPENDIX}

As an alternative approach, the texture-estimated misorientation distribution function is derived in Bunge's formalism. From Eq. (13),

$$
M^{\prime}(\Delta g)=\oint f(g) f(\Delta g \cdot g) d g=\oint f^{*}(g) f(\Delta g \cdot g) d g
$$

From Eq. (4.7) of Bunge (1982), Eq. (A1) is equivalent to

$$
\begin{aligned}
M^{\prime}(\Delta g)=\sum_{l=0}^{\infty} \sum_{u=1}^{M(l)} \sum_{v=1}^{N(l)} \sum_{l^{\prime}=0}^{\infty} \sum_{u^{\prime}=1}^{M^{\prime}\left(l^{\prime}\right)} \sum_{v^{\prime}=1}^{N^{\prime}\left(l^{\prime}\right)} \\
\times C_{l}^{* u v} C_{l^{\prime}}^{u^{\prime} v^{\prime}} \oint_{\tilde{T}_{l}^{*}}^{* u v}(g) \dot{T}_{l^{\prime}}^{u^{\prime} v^{\prime}}(\Delta g \cdot g) d g,
\end{aligned}
$$




$$
\dot{T}_{l}^{* u v}(g)=\sum_{\alpha=-l}^{1} \dot{A}_{l}^{\alpha u} \dot{T}_{l}^{* \alpha v}(g)
$$

and from the addition theorem,

$$
\dot{T}_{l^{\prime}}^{u^{\prime} v^{\prime}}(\Delta g \cdot g)=\sum_{s=-l^{\prime}}^{l^{\prime}} \dot{T}_{l^{\prime}}^{u^{\prime} s}(\Delta g) \dot{T}_{l^{\prime}}^{s v^{\prime}}(g)
$$

From the orthogonality relation,

$$
\oint T_{l}^{\alpha v}(g) \dot{T}_{l^{\prime}}^{s \prime^{\prime}}(g) d g=\frac{1}{2 l+1} \delta_{l l^{\prime}} \delta_{\alpha s} \delta_{v v^{\prime}}
$$

and the symmetry relation,

$$
\sum_{\alpha=-l}^{l} \dot{A}_{l}^{\alpha u} \tilde{T}_{l}^{u^{\prime} \alpha}(\Delta g)=\tilde{T}_{l}^{u^{\prime} u}(\Delta g)
$$

it follows that

where

$$
M^{\prime}(\Delta g)=\sum_{l=0}^{\infty} \sum_{u=1}^{M(l)} \sum_{u^{\prime}=1}^{M^{\prime}\left(l^{\prime}\right)} \hat{C}_{l}^{u u^{\prime}} T_{l}^{u^{\prime} u}(\Delta g),
$$

$$
\hat{C}_{l}^{u u^{\prime}}=\frac{1}{2 l+1} \sum_{v=1}^{N(l)} C_{l}^{* u v} C_{l}^{u^{\prime} v} .
$$

Equation (A7) is equivalent to (18) in the body of the paper, but in Bunge's formalism. 